\title{
Predicting Macrophage Activation Syndrome in Childhood- onset Systemic Lupus Erythematosus Patients at Diagnosis
}

\author{
Maya Gerstein ${ }^{1}(\mathbb{D})$, R. Ezequiel Borgia ${ }^{2}$ (D) Daniela Dominguez ${ }^{3}$ (D), Brian M. Feldman ${ }^{3}$ (D), \\ Fangming Liao ${ }^{3}$, Deborah M. Levy ${ }^{3}$ (D) Lawrence $\mathrm{Ng}^{3}$, Mohamed Abdelhaleem ${ }^{4}$ (D), \\ Earl D. Silverman ${ }^{3}$ (D) and Linda T. Hiraki ${ }^{3}$ (D)
}

\begin{abstract}
Objective. Macrophage activation syndrome (MAS), a life-threatening inflammatory complication, is increasingly recognized in childhood-onset systemic lupus erythematosus (cSLE). It can be a challenge to differentiate active cSLE from MAS. We generated decision rules for discriminating MAS from active cSLE in newly diagnosed patients.

Methods. We conducted a retrospective cohort study of consecutive, newly diagnosed, active cSLE patients with fever, requiring hospital admission to The Hospital for Sick Children from January 2003 to December 2007 (cohort 1) and January 2008 to December 2013 (cohort 2). All patients met $\geq 4$ American College of Rheumatology or Systemic Lupus International Collaborating Clinics criteria, and were steroid-naïve and infection-free. MAS was diagnosed based on expert opinion. Recursive partitioning was applied to each cohort to derive a decision rule based on clinical and laboratory features, distinguishing MAS from non-MAS cSLE. Each decision rule was applied to the alternate, independent cohort. Sensitivity and specificity of these decision rules were compared to existing criteria.

Results. Cohort $1(\mathrm{n}=34)$ and cohort $2(\mathrm{n}=41)$ each had 10 patients with MAS. Recursive partitioning in cohort 1 identified ferritin $\geq 699 \mu \mathrm{g} / \mathrm{L}$ as the sole best discriminator between MAS and non-MAS patients $\left(\mathrm{R}^{2}=0.48\right)$, and in cohort 2 , ferritin $\geq 1107 \mu \mathrm{g} / \mathrm{L}$ was the best discriminator for MAS, followed by lymphocytes $<0.72 \times 10^{3} / \mathrm{mm}^{3}\left(\mathrm{R}^{2}=0.52\right)$. Cross-validation of our decision rules maintained $90-100 \%$ sensitivity and $65-85 \%$ specificity.

Conclusion. Our decision rule demonstrated improved performance compared to preliminary guidelines for MAS in cSLE from the Lupus Working Group of the Paediatric Rheumatology European Society and familial hemophagocytic lymphohistiocytosis diagnostic criteria. Validation in independent cohorts is required.
\end{abstract}

Key Indexing Terms: macrophage activation syndrome, pediatric systemic lupus erythematosus, systemic lupus erythematosus

Macrophage activation syndrome (MAS) is a potentially life-threatening complication of inflammatory disorders

LTH is supported by a grant from the Arthritis Society (TAS).

1M. Gerstein, MD, Division of Rheumatology, The Hospital for Sick Children, Toronto, Ontario, Canada, and Pediatric Rheumatology Unit, Edmond and Lily Safra Children's Hospital, Sheba Medical Center, Tel Hashomer, Ramat Gan, Israel; 2R.E. Borgia, MD, Division of Rheumatology, The Hospital for Sick Children, Toronto, and Department of Pediatrics, College of Medicine, University of Florida, Gainesville, Florida, USA; 3D. Dominguez, MD, MSc, B.M. Feldman, MD, MSc, FRCPC, F. Liao, MSc, D.M. Levy, MD, MS, FRCPC, L. Ng, BSc, E.D. Silverman, MD, FRCPC, L.T. Hiraki, MD, FRCPC, ScD, Division of Rheumatology, The Hospital for Sick Children, Toronto, Ontario Canada; 4M. Abdelhaleem, MBBCh, PhD, FRCPC, Division of Haematopathology, The Hospital for Sick Children, Toronto, Ontario, Canada.

The authors declare no conflicts of interest relevant to this article.

Address correspondence to Dr. L.T. Hiraki, Clinician Scientist, Division of Rheumatology, Research Institute, The Hospital for Sick Children, PGCRL, 686 Bay Street, Toronto, ON M5G 0A4, Canada.

Email: linda.hiraki@sickkids.ca.

Accepted for publication November 20, 2020. including childhood-onset systemic lupus erythematosus (cSLE) and other pediatric and adult rheumatic diseases. ${ }^{1,2,3,4}$ MAS, a secondary form of familial hemophagocytic histiocytosis (fHLH), is so named for the marked clinical and laboratory similarity of the diseases. Both MAS and fHLH are characterized by excessive activation of differentiated macrophages with the resultant presence of hemophagocytic macrophages in the bone marrow, liver, spleen, and/or lymph nodes. These activated macrophages phagocytose multiple hematopoietic lineages, contributing to pancytopenia, which is exacerbated by systemic inflammatory responses. ${ }^{5,6}$ Other features include coagulopathy, hypertriglyceridemia, hypofibrinogenemia, and hyperferritinemia. ${ }^{7}$ The clinical presentation manifests with persistent fever, hepatosplenomegaly, lymphadenopathy, and central nervous system (CNS) dysfunction. ${ }^{8}$

The clinical features of active SLE include many of the clinical features of MAS, and, in particular, patients with SLE can present with hepatosplenomegaly, lymphadenopathy, and CNS dysfunction. Similarly, the characteristic laboratory features of MAS, including pancytopenia, coagulopathy, hypertriglyceridemia, and hyperferritinemia, are seen in active SLE. ${ }^{9,10,11}$ Due to the overlapping features, it can be difficult to differentiate active 
SLE from MAS., ${ }^{2,12,13,14}$ In 2009, the Lupus Working Group of the Paediatric Rheumatology European Society (PReS) developed preliminary guidelines for the diagnosis of MAS in cSLE, but these have not been validated. ${ }^{3}$

The purpose of this study was to develop a decision rule to differentiate MAS from active cSLE among newly diagnosed, treatment-naive patients with cSLE. Our second aim was to compare the performance of our decision rules (sensitivity and specificity) with those from the PReS Lupus Working Group preliminary criteria for MAS in cSLE and the $2004 \mathrm{fHLH}$ diagnostic criteria. ${ }^{3,15}$

\section{METHODS}

Patient population. We restricted our study population from our Lupus Clinic database to include patients admitted to The Hospital for Sick Children (SickKids), Toronto, for newly diagnosed cSLE between January 2003 to December 2007 (cohort 1), and January 2008 to December 2013 (cohort 2). We extracted prospectively collected clinical and laboratory data. All patients met $\geq 4$ American College of Rheumatology and/or Systemic Lupus International Collaborating Clinics classification criteria for SLE. ${ }^{16,17}$ We reviewed all hospital admissions lasting a minimum of 3 days, and occurring within 2 months prior to, and up to, 12 months following cSLE diagnosis. Among the admitted patients, we identified those with a clinical diagnosis of MAS by the treating pediatric rheumatologist during the admission (expert opinion). Patients were excluded for (1) absence of fever, (2) prior steroid use at time of presentation and diagnosis, (3) isolated infection, or (4) elective admission for treatment or procedures. Institutional Research Ethics Board (REB) approval was obtained prior to initiation of the study (REB \#1000035186).

Clinical and laboratory variables. We reviewed the clinical and laboratory features of all patients included in our cohort, during hospital admission. Clinical features of MAS included documentation of fever $\left(38.5^{\circ} \mathrm{C}\right.$ or higher), CNS dysfunction (irritability, seizures, severe headache, hallucinations, disorientation, or coma), splenomegaly, hepatomegaly, and hemorrhagic manifestations (purpura, easy bruising, or mucosal bleeding). Laboratory variables included complete blood count and differential (hemoglobin, white blood cell [WBC], neutrophil, lymphocyte, and platelet counts), direct Coombs/direct antiglobulin test, triglycerides, aspartate aminotransferase, alanine aminotransferase, lactate dehydrogenase, albumin, ferritin, fibrinogen, low-density lipoprotein, high-density lipoprotein, triglycerides, international normalized ratio, activated partial thrombin time, D-dimer, C3, C4, IgG, urea, serum creatinine, erythrocyte sedimentation rate (ESR), sodium, calcium, and C-reactive protein. The prevalence of SLE features was compared between MAS and non-MAS patients within each cohort using Fisher exact test and a significance threshold adjusted for multiple comparisons $(P<0.003)$.

When available, bone marrow aspirates/biopsies were reviewed for evidence of hemophagocytosis. Additional MAS markers, soluble CD25 (sCD25/soluble interleukin 2 receptor $\alpha$ chain), CD163, and natural killer (NK) cell activity were measured in 4 patients. Since bone marrow aspirates/ biopsies and these additional MAS markers were not tested in all participants, they were excluded factors in recursive partitioning. When testing the performance of the $\mathrm{fHLH}$ criteria in our population, we restricted to patients with bone marrow aspirates/biopsies and did not include features of sCD25 and NK cell activity.

Statistical analysis. Within each cohort (cohort 1: 2003-2007, cohort 2: 2008-2013), patients were assigned to 1 of 2 mutually exclusive groups: (a) MAS and (b) non-MAS. We performed recursive partitioning in each cohort using baseline quantitative laboratory measures and binary clinical features to derive a decision rule for identifying MAS (RStudio 0.99.902, RStudio Team). ${ }^{18}$ To maximize information and avoid overfitting, we compared parameters of fit (area under the receiver-operating characteristic [ROC] and $\mathrm{R}^{2}$ ) with complexity and cross-validation error. We tested the performance of each decision rule by applying the rule to the alternate, independent cohort (i.e., cohort 1 derived decision rule was applied to cohort 2 and vice versa). We calculated the sensitivity and specificity of the decision rule for identifying MAS in each cohort. Last, we completed recursive partitioning on the total patient population to increase the sample size and power of our analysis. The sensitivity and specificity of the existing primary fHLH and the PReS Lupus Working Group preliminary criteria for MAS in cSLE were determined as applied to our study cohort. In sensitivity analyses, we added the ratio of baseline serum ferritin $(\mathrm{ng} / \mathrm{mL})$ to erythrocyte sedimentation rate $(\mathrm{mm} / \mathrm{h})$ to the baseline laboratory and clinical variables and regenerated the decision rules.

\section{RESULTS}

We reviewed 406 newly diagnosed cSLE patient charts, of which 214 patients had at least 1 hospital admission. After omitting 138 patients based on our exclusion criteria, our study cohort included 34 patients in cohort 1 (10 with MAS and 24 without MAS) and 41 in cohort (10 with MAS and 31 without MAS). In $99 \%$ of patients, hospital admission preceded or coincided with SLE diagnosis, with only 1 patient requiring admission 28 days after diagnosis. All patients were naïve to corticosteroid treatment prior to presentation.

The mean age at hospital admission was 14.0 (SD 2.5) years in cohort 1 and $13.6(\mathrm{SD} 2.5)$ years in cohort $2(P=0.52)$. The majority of patients were female (cohort 1: $85 \%$, cohort 2: $81 \%$, $P=0.76$; Table 1). The prevalence of specific SLE manifestations were comparable in cohorts 1 and 2 .

Regarding MAS manifestations, none of the clinical features typically associated with MAS was found to be significantly different between the MAS and non-MAS groups (Table 2). Bone marrow (BM) aspirate and/or biopsies were performed on 43 patients (20 in cohort 1 and 23 in cohort 2 ). In cohort 1 , significant hemophagocytosis was present in 3/6 MAS patients, and $3 / 14$ non-MAS patients who had BM aspiration and biopsy (50\% vs $21 \%, P=0.30$ ). In cohort 2 , hemophagocytosis was evident on 2 of the 9 MAS patient BM specimens, and 1 of the 14 non-MAS specimens ( $22 \%$ vs $7 \%, P=0.54$ ).

Recursive partitioning. We used recursive partitioning to determine cut-off values for laboratory tests differentiating patients with MAS from patients without MAS in each cohort (Figure 1). At the outset, the pretest probability of a patient having MAS was $27 \%$ (20/75 of our total cohort had MAS). In cohort 1 , recursive threshold testing identified ferritin as the sole variable differentiating MAS vs non-MAS patients, with a cut-off value of $\geq 699 \mu \mathrm{g} / \mathrm{L}\left(\mathrm{R}^{2}=0.48\right)$. Testing this decision rule in cohort 2 demonstrated a sensitivity of $90 \%$ and a specificity of $81 \%$ for MAS (area under the receiver-operating characteristic [AUC] $=0.87$ (Supplementary Figure 1A, available with the online version of this article).

When we performed recursive partitioning in cohort 2 (Figure 2, including hierarchy of testing), we derived a decision rule that best differentiated MAS from non-MAS patients and identified the following thresholds: (1) ferritin $\geq 1107 \mu \mathrm{g} / \mathrm{L}$, and (2) lymphocytes $<0.72 \times 10^{3} / \mathrm{mm}^{3}$, with a $\mathrm{R}^{2}=0.52$. When we tested this decision rule in cohort 1 , the sensitivity was $90 \%$ 
Table 1. Systemic lupus erythematosus features at diagnosis.

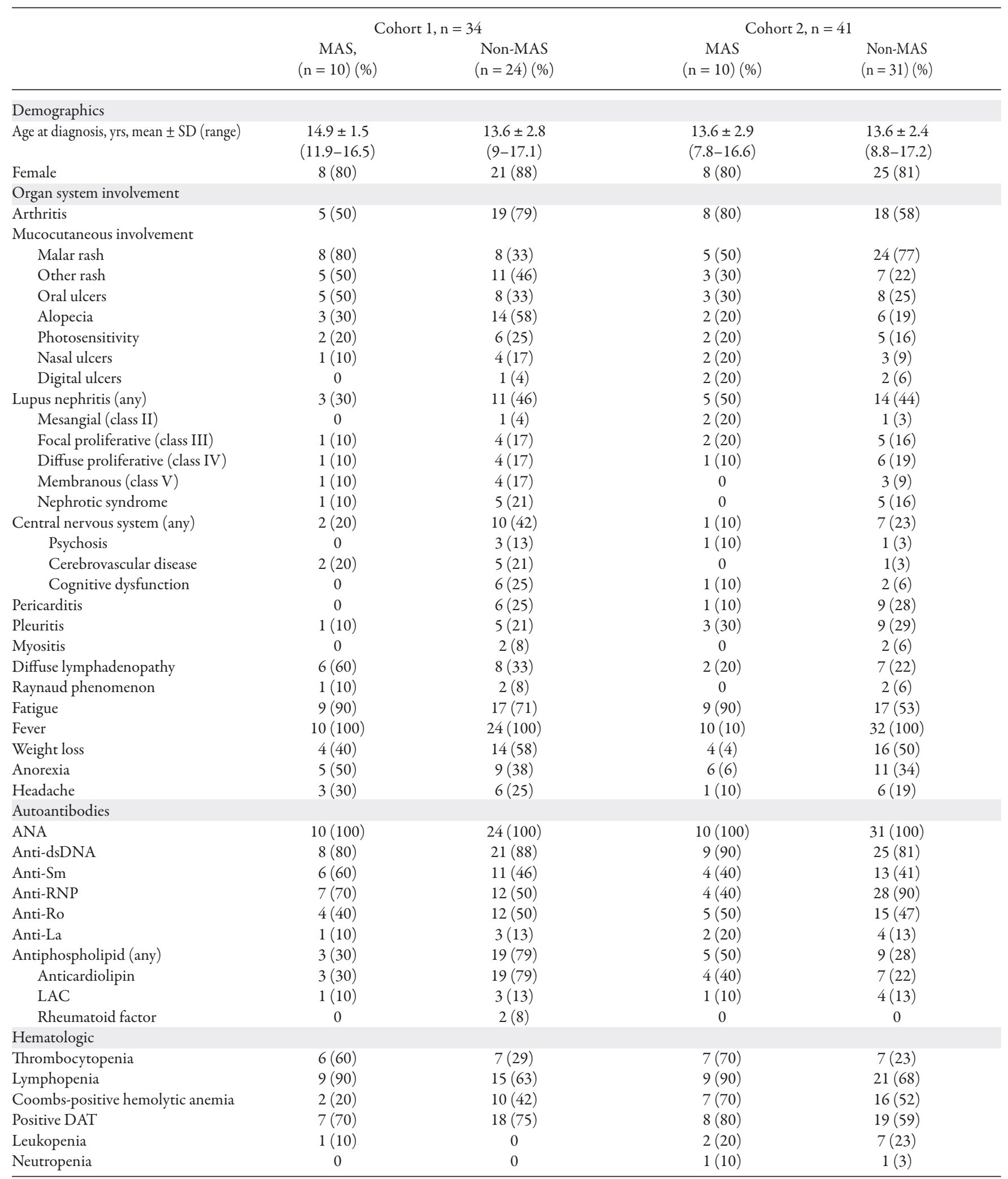

Values are expressed as n (\%) unless otherwise indicated. ANA: antinuclear antibody; DAT: direct antiglobulin test; LAC: lupus anticoagulant; MAS: macrophage activation syndrome. 
Table 2. Hemophagocytic lymphohistiocytosis clinical features and laboratory results in MAS and non-MAS patients by cohort.

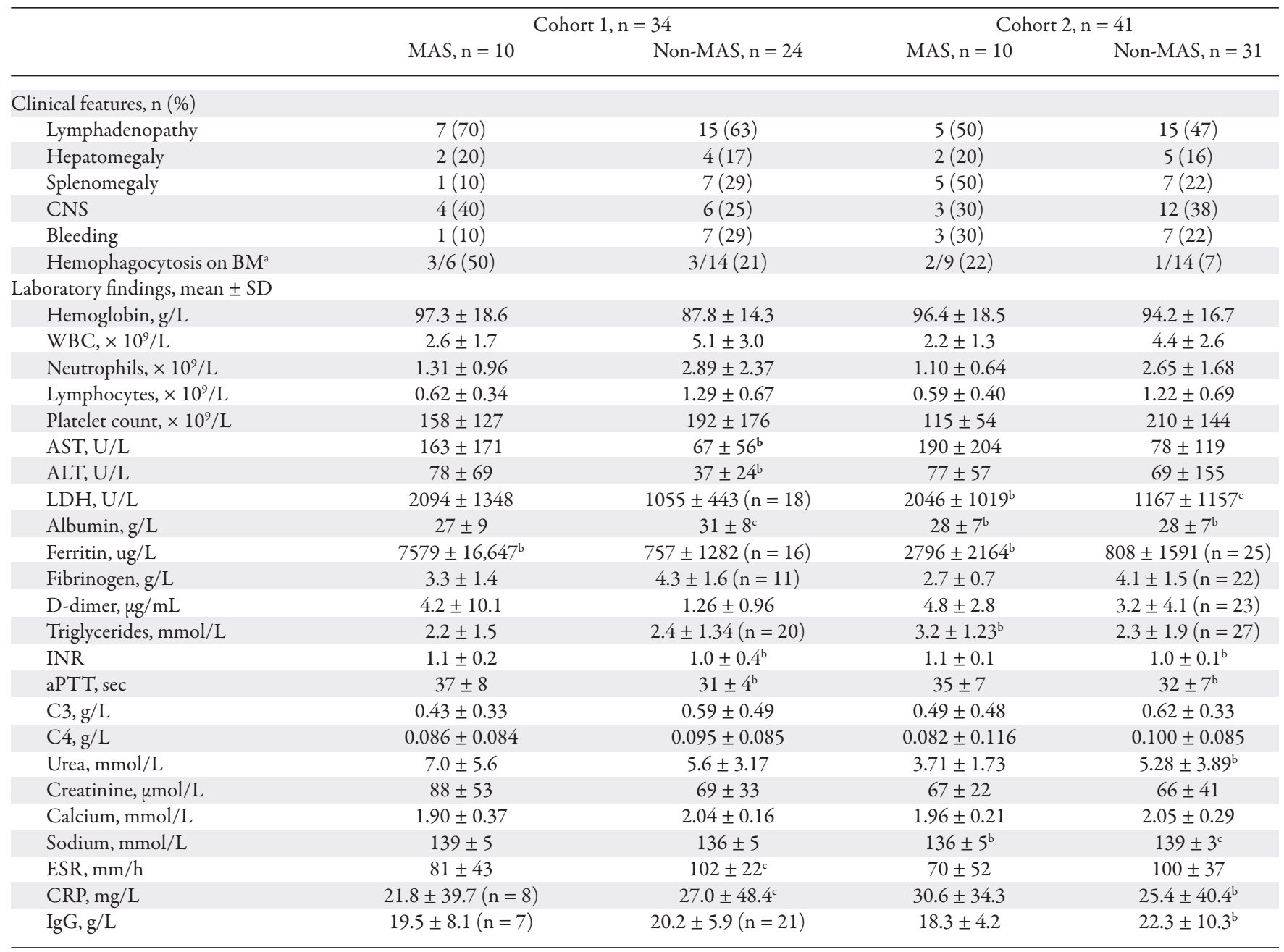

${ }^{a} \mathrm{BM}$ available in a subset of patients. Number reported in each group. ${ }^{\mathbf{b}}$ Laboratory data missing on 1 subject. ${ }^{\mathbf{c}}$ Laboratory data missing on 2 subjects. ALT: alanine aminotransferase; aPTT: activated partial thromboplastin time; AST: aspartate aminotransferase; BM: bone barrow; CNS: central nervous system; CRP: C-reactive protein; ESR: erythrocyte sedimentation rate; LDH: lactate dehydrogenase; INR: international normalized ratio; MAS: macrophage activation syndrome; WBC: white blood cell.

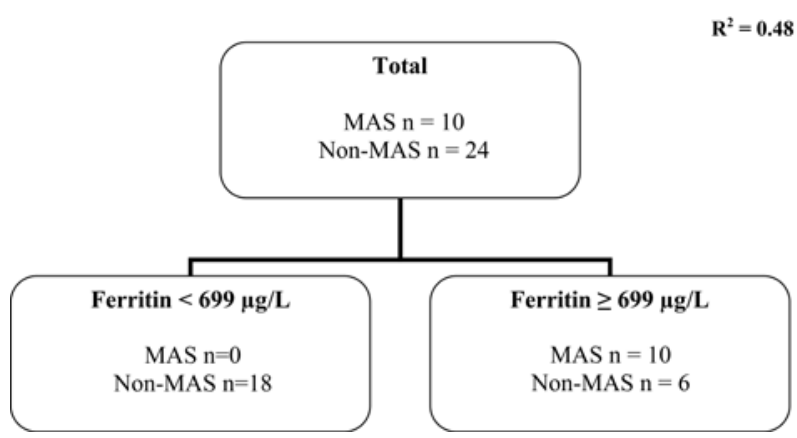

Figure 1. Recursive partitioning decision rule derived from cohort 1 . The boxes summarize the numbers of MAS and non-MAS patients in cohort 1 , and the decision node of ferritin threshold $699 \mathrm{ug} / \mathrm{mL}$, terminal branches, and respective sample sizes. The $\mathrm{R}^{2}$ of 0.48 reflects the decision rule fit in cohort 1. MAS: macrophage activation syndrome. and specificity of $63 \%$ (AUC $=0.77$; Supplementary Figure 1B, available with the online version of this article).

We next combined the 2 cohorts into a single cohort and generated a third decision rule for differentiating patients with MAS from patients without MAS (Figure 3, including hierarchy of testing). Serial cut-off testing resulted in the following algorithm: (1) ferritin $>699 \mu \mathrm{g} / \mathrm{L},(2) \mathrm{WBC}<2.3 \times 10^{3} / \mathrm{mm}^{3}$, which resulted in $\mathrm{R}^{2}=0.62$.

In testing the performance of fHLH criteria restricted to patients with $\mathrm{BM}$ aspirates/biopsies, we observed that in cohort $1,3 / 6$ patients with MAS and 2/14 without MAS met $>5$ criteria for $\mathrm{fHLH}^{15}$ (Table 3; Supplementary Table 1, available with the online version of this article). This resulted in a sensitivity of $50 \%$ and specificity of $86 \%$. In cohort 2, 4/9 patients with MAS and none of the non-MAS met $>5$ criteria for $\mathrm{fHLH}$. This resulted 


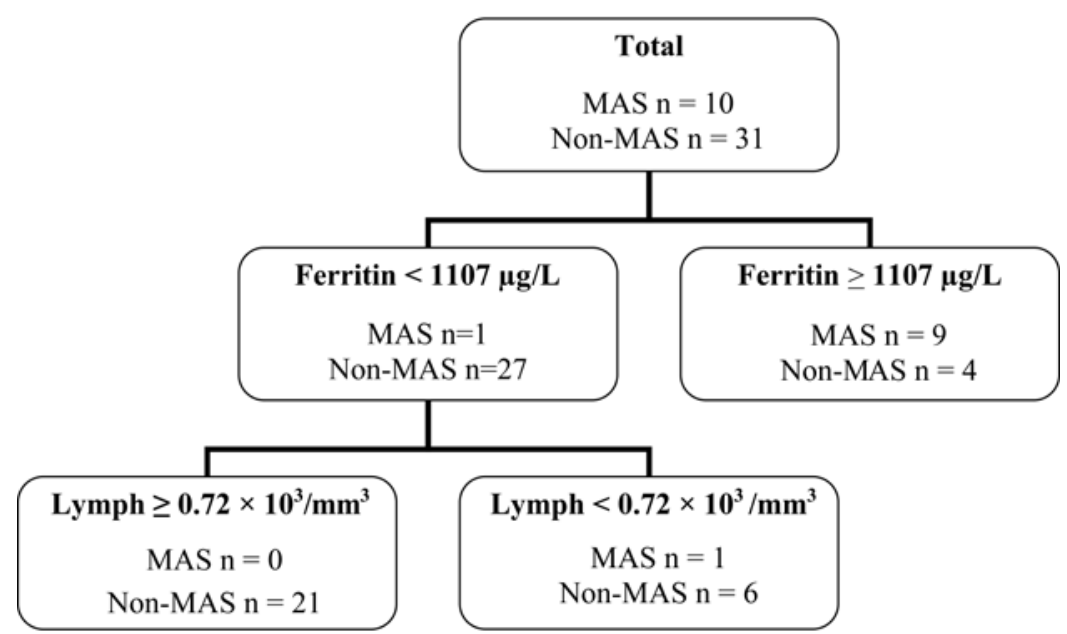

Figure 2. Recursive partitioning decision rule derived from cohort 2. The boxes summarize the numbers of MAS and non-MAS patients in cohort 2, and the decision node of ferritin threshold $1107 \mu \mathrm{g} / \mathrm{mL}$, followed by lymphocyte threshold count of $0.72 \times 10^{3} / \mathrm{mm}^{3}$, terminal branches, and respective sample sizes. The $\mathrm{R}^{2}$ of 0.52 reflects the decision rule fit in cohort 2 . Lymph: lymphocyte; MAS: macrophage activation syndrome.

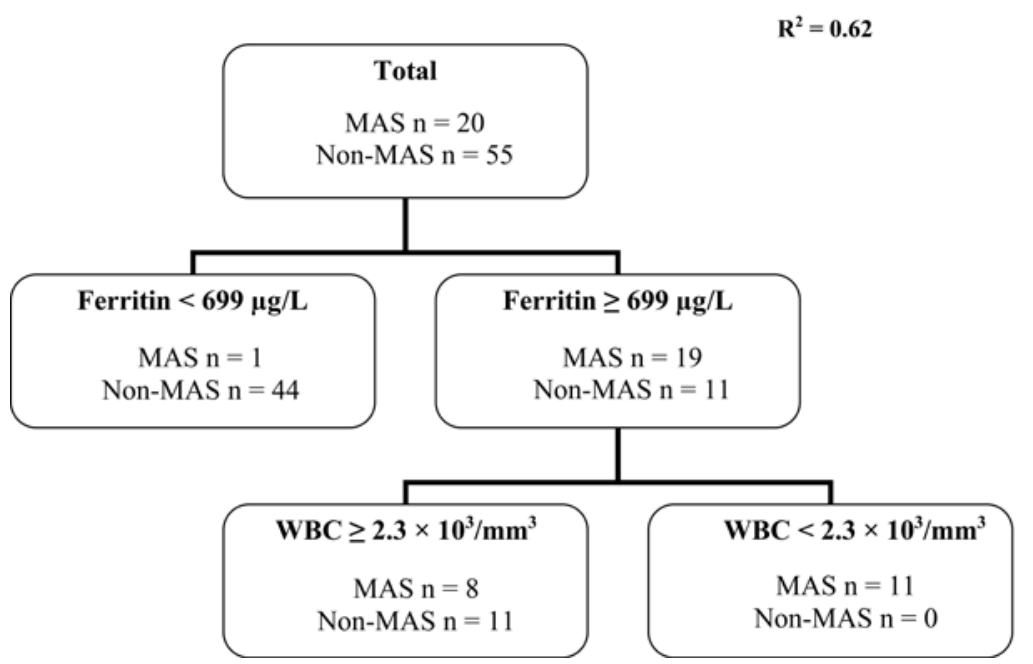

Figure 3. Recursive partitioning decision rule derived for both cohorts. The boxes summarize the numbers of MAS and non-MAS patients in the total cohort, and the decision node of ferritin threshold $699 \mathrm{ug} / \mathrm{mL}$, followed by WBC threshold count of $2.3 \times 10^{3} / \mathrm{mm}^{3}$, terminal branches, and respective sample sizes. The $\mathrm{R}^{2}$ of 0.62 reflects the decision rule fit in the total cohort. MAS: macrophage activation syndrome; WBC: white blood cell.

in a sensitivity of $44 \%$ and specificity of $100 \%$. In the combined cohort 1 and 2,7/15 patients with MAS and 2/28 of the non-MAS patients met fHLH criteria, which resulted in a sensitivity of $47 \%$ and a specificity of $93 \%$ (Table 3). Testing the PReS Lupus Working Group preliminary criteria for MAS in cSLE in cohort 1 demonstrated a sensitivity of $100 \%$ and specificity of $17 \%$, and in cohort 2 a sensitivity of $100 \%$ and specificity of $32 \%$. In the combined cohort, all 20 patients with MAS and 41/55 non-MAS met the PReS Lupus Working Group preliminary criteria for MAS in cSLE, resulting in a sensitivity of $100 \%$ and a specificity of $25 \%$.

Sensitivity analyses adding the baseline serum ferritin/ESR ratio did not change the resultant decision rule for cohorts 1 or 2 , nor in the combined cohort.

\section{DISCUSSION}

MAS is a life-threatening complication of SLE that is difficult to differentiate from active SLE without MAS, due to overlapping clinical and laboratory manifestations. ${ }^{4,13,19}$ We developed criteria distinguishing MAS in patients with cSLE from those with active SLE alone, using recursive partitioning, in 2 independent cohorts of treatment-naïve patients with cSLE. Our new criteria demonstrated improved discriminatory power compared 
Table 3. Performance of existing criteria for HLH and MAS diagnosis.

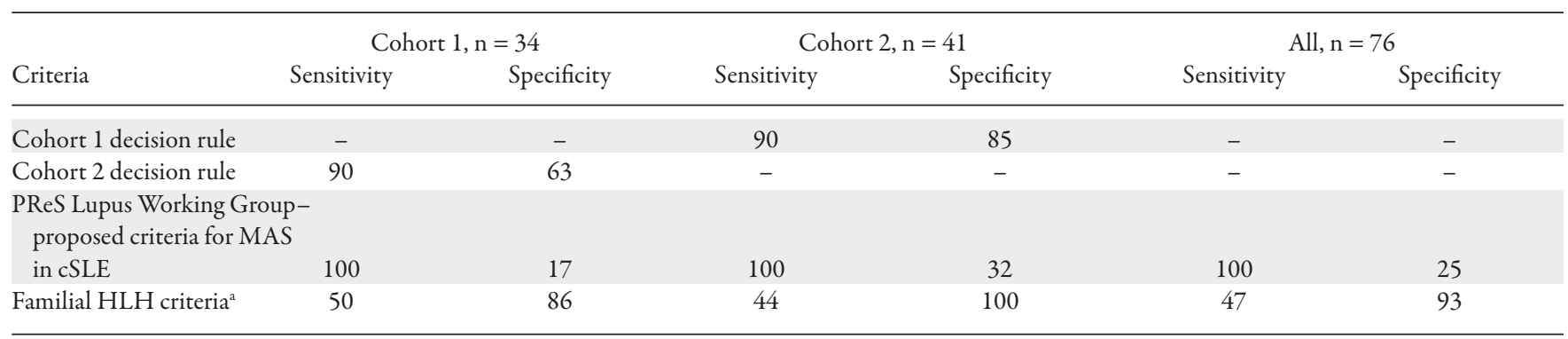

- Estimation of sensitivity and specificity in these cohorts would be overfit, since these cohorts gave rise to the decision rule. ${ }^{a}$ Restricted to patients with bone marrow aspirates/biopsies (cohort $1: \mathrm{n}=20$; cohort $2: \mathrm{n}=23$ ). Soluble interleukin receptor and natural killer cell activity were not included in sensitivity and specificity calculations. cSLE: childhood-onset systemic lupus erythematosus; HLH: hemophagocytic lymphohistiocytosis; MAS: macrophage activation syndrome; PReS: Paediatric Rheumatology European Society.

to existing diagnostic criteria for $\mathrm{fHLH}^{15}$ and the PReS Lupus Working Group preliminary criteria for MAS in cSLE. ${ }^{3}$

We found the $\mathrm{FHLH}$ diagnostic criteria had poor sensitivity for diagnosing MAS secondary to SLE, likely due to limited availability of special tests such as NK cell activity, or the extreme thresholds for laboratory abnormalities such as cytopenias and ferritin levels. Another limitation of applying the existing fHLH criteria to patients with cSLE is the central role of BM biopsy for fHLH diagnosis. ${ }^{15} \mathrm{BM}$ aspirate or biopsy offers limited utility for MAS diagnosis in rheumatic diseases. As such, preliminary guidelines for MAS in cSLE do not require BM examination., We demonstrated that the presence of hemophagocytosis on BM aspirate/biopsy is neither sensitive nor specific for the diagnosis of secondary MAS in patients with $\mathrm{cSLE}^{20}$; this is consistent with observations in $\mathrm{fHLH}^{20}$ and other autoimmune diseases. ${ }^{1,4}$ Requiring a BM specimen for MAS diagnosis will likely delay diagnosis and increase the mortality associated with MAS.

Recognizing the limited sensitivity of fHLH diagnostic criteria for identifying MAS in patients with cSLE, the PReS Lupus Working Group proposed guidelines for the diagnosis of MAS in cSLE that include many features SLE patients manifest at the time of diagnosis. ${ }^{3}$ Although these PReS guidelines had improved sensitivity compared to the fHLH diagnostic criteria for MAS in SLE, they were also less specific than fHLH criteria when applied to our inpatient cohorts, since fever, ${ }^{10,21}$ splenomegaly, ${ }^{10}$ cytopenias (> 2/3 lineages) ${ }^{10}$ hypertriglyceridemia, ${ }^{22}$ hemophagocytosis in $\mathrm{BM}^{20}{ }^{20}$ low $\mathrm{NK}$ cell activity, ${ }^{23}$ elevated ferritin, ${ }^{11}$ and elevated sCD25 levels ${ }^{24,25}$ are frequently seen in patients with active SLE.

Since concern for MAS often arises in hospitalized SLE patients, we aimed to develop MAS criteria with improved power to discriminate MAS from active SLE, over existing fHLH and PReS Lupus Working Group criteria. We restricted our cohort to patients admitted to hospital with documented fever and no prior exposure to corticosteroids. This selection strategy not only ensured that our MAS and non-MAS case-control populations represented real-life clinical scenarios, but that laboratory variables were unaffected by past medication exposures. When we applied the PReS Lupus Working Group criteria for MAS in SLE to our cohort, we found that $100 \%$ of the cSLE with MAS patients met the criteria. However, there was a high false positive rate as well, with $75 \%$ of the newly diagnosed cSLE patients without the clinical diagnosis of MAS also meeting the criteria.

We created 3 decision rules for MAS in cSLE, using recursive partitioning, 1 rule from each cohort, and a third derived from both cohorts together. Recursive partitioning used the cohort data to create the best fitting model, which in this case demonstrated an $\mathrm{R}^{2}$ value of $0.48,0.52$, and 0.62 (in cohorts 1 , 2 , and cohorts 1 and 2 combined, respectively). However, there was a risk of overfitting a model and generating an algorithm specific to the dataset from which it arose. Hence, the true test of the model's performance is in its application to an independent cohort. Our study was designed specifically to overcome this obstacle, by having 2 cohorts derived from the same population, and being managed by the same experts and laboratories. Therefore, we were able to apply each cohort's rule on the independent parallel cohort, using it as a testing cohort.

Our proposed criteria for MAS in cSLE are similar to those proposed by the PReS Lupus Working Group. All 3 rules identified ferritin as the first variable distinguishing between MAS and non-MAS among newly diagnosed, treatment-naïve patients with cSLE. However, our threshold for hyperferritinemia was higher than the one proposed by the PReS Lupus Working Group ( $\geq 699 \mu \mathrm{g} / \mathrm{L}$ compared with $>500 \mu \mathrm{g} / \mathrm{L}$ ). As with the PReS criteria, we also found that cytopenia was informative, specifically lymphopenia (cohort 2 decision rule) and leukopenia (total cohort decision rule) as the second most informative discriminators between MAS and non-MAS. A prior study demonstrated improved sensitivity and specificity of serum ferritin/ESR ratio for diagnosing MAS in systemic juvenile idiopathic arthritis populations, over ferritin alone. ${ }^{26}$ Our sensitivity analyses added the baseline ferritin/ESR ratio to our recursive partitioning models. However, we did not observe improved discriminatory power for MAS in cSLE, of the ferritin/ESR ratio over ferritin and cytopenias.

Our proposed MAS criteria in cSLE identified the same informative laboratory variables as the PReS Lupus Working Group. However, the more extreme thresholds we propose improved the specificity of our criteria over those proposed by PReS Lupus Working Group. In clinical application, it may be appropriate to consider a sequential application of criteria, beginning with the most sensitive criteria from the Lupus Working Group of PReS 
to ensure complete identification of all MAS cases, followed by more specific criteria proposed by our work, to reduce the number of false positive MAS cases.

Our study findings should be considered in light of some potential limitations. There is no diagnostic gold standard for MAS, which necessitated the reliance on pediatric rheumatologist diagnosis. In our study, each MAS diagnosis was independently verified by an investigator, who reviewed the entire disease course in hospital including therapy response. Any disputed diagnoses were discussed and validated by all the investigators. ${ }^{27}$ Also, we did not have access to a third independent cohort in which to test our final criteria's performance. Therefore, we concluded that these proposed criteria should be validated.

Our study had a number of strengths. We were able to focus on a large number of steroid-naïve, acutely ill cSLE patients at disease presentation, in 2 independent cohorts separated by era. In this way, our study cohorts represented an ill cSLE population, in whom MAS diagnostic criteria would have the greatest clinical benefit. Making an early and timely diagnosis of MAS is critical, since the therapy differs for MAS and active SLE disease. Our study also demonstrated an increased prevalence of MAS (26\%) among our selected cohort of cSLE patients requiring hospitalization. This is compared to our prior work that reported a MAS prevalence of $9 \%$ in our expanded cSLE population. ${ }^{27}$ This higher MAS prevalence in hospitalized cSLE patients emphasizes the importance of considering MAS as a complicating disease process in SLE patients requiring hospitalization, rather than attributing illness to SLE disease activity alone.

Our proposed criteria for diagnosing MAS in patients with cSLE identified ferritin, lymphocyte, and leukocyte counts as the most informative factors in discriminating MAS from active SLE. Our criteria have demonstrated improved sensitivity and specificity for MAS in our cohorts when compared to current diagnostic criteria for fHLH and the PReS Lupus Working Group preliminary guidelines for MAS in cSLE. Testing our criteria in independent cSLE and adult-onset SLE cohorts would provide additional value for the generalizability and utility of our proposed criteria.

\section{ONLINE SUPPLEMENT}

Supplementary material accompanies the online version of this article.

\section{REFERENCES}

1. Kumakura S, Ishikura H, Kondo M, Murakawa Y, Masuda J, Kobayashi S. Autoimmune-associated hemophagocytic syndrome. Mod Rheumatol 2004;14:205-15.

2. Avcin T, Tse SML, Schneider R, Ngan B, Silverman ED. Macrophage activation syndrome as the presenting manifestation of rheumatic diseases in childhood. J Pediatr 2006;148:683-6.

3. Parodi A, Davi S, Pringe AB, Pistorio A, Ruperto N, Magni-Manzoni S, et al. Macrophage activation syndrome in juvenile systemic lupus erythematosus: a multinational multicenter study of thirty-eight patients. Arthritis Rheum 2009;60:3388-99.

4. Carvalheiras G, Anjo D, Mendonça T, Vasconcelos C, Farinha F. Hemophagocytic syndrome as one of the main primary manifestations in acute systemic lupus erythematosus--case report and literature review. Lupus 2010;19:756-61.

5. Canna SW, Wrobel J, Chu N, Kreiger PA, Paessler M, Behrens EM. Interferon- $\gamma$ mediates anemia but is dispensable for fulminant toll-like receptor 9-induced macrophage activation syndrome and hemophagocytosis in mice. Arthritis Rheum 2013;65:1764-75.

6. Behrens EM, Canna SW, Slade K, Rao S, Kreiger PA, Paessler M, et al. Repeated TLR9 stimulation results in macrophage activation syndrome-like disease in mice. J Clin Invest 2011;121:2264-77.

7. Imashuku S, Hyakuna N, Funabiki T, Ikuta K, Sako M, Iwai A, et al. Low natural killer activity and central nervous system disease as a high-risk prognostic indicator in young patients with hemophagocytic lymphohistiocytosis. Cancer 2002;94: 3023-31.

8. Ravelli A, Magni-Manzoni S, Pistorio A, Besana C, Foti T, Ruperto $\mathrm{N}$, et al. Preliminary diagnostic guidelines for macrophage activation syndrome complicating systemic juvenile idiopathic arthritis. J Pediatr 2005;146:598-604.

9. Pons-Estel BA, Catoggio LJ, Cardiel MH, Soriano ER, Gentiletti S, Villa AR, et al. The GLADEL multinational Latin American prospective inception cohort of 1,214 patients with systemic lupus erythematosus: ethnic and disease heterogeneity among "Hispanics." Medicine 2004;83:1-17.

10. Hiraki LT, Benseler SM, Tyrrell PN, Hebert D, Harvey E, Silverman ED. Clinical and laboratory characteristics and long-term outcome of pediatric systemic lupus erythematosus: a longitudinal study. J Pediatr 2008;152:550-6.

11. Zandman-Goddard G, Shoenfeld Y. Ferritin in autoimmune diseases. Autoimmun Rev 2007;6:457-63.

12. Qian J, Yang CD. Hemophagocytic syndrome as one of main manifestations in untreated systemic lupus erythematosus: two case reports and literature review. Clin Rheumatol 2007;26:807-10.

13. Yeap ST, Sheen JM, Kuo HC, Hwang KP, Yang KD, Yu HR. Macrophage activation syndrome as initial presentation of systemic lupus erythematosus. Pediatr Neonatol 2008;49:39-42.

14. Kwon CM, Jung YW, Yun DY, Kim HD, Cho HS, Hong YH, et al. A case of acute pericarditis with hemophagocytic syndrome, cytomegalovirus infection and systemic lupus erythematosus. Rheumatol Int 2008;28:271-3.

15. Henter JI, Horne A, Aricó M, Egeler RM, Filipovich AH, Imashuku S, et al. HLH-2004: Diagnostic and therapeutic guidelines for hemophagocytic lymphohistiocytosis. Pediatr Blood Cancer 2007;48:124-31.

16. Petri M, Orbai AM, Alarcón GS, Gordon C, Merrill JT, Fortin PR, et al. Derivation and validation of the Systemic Lupus International Collaborating Clinics classification criteria for systemic lupus erythematosus. Arthritis Rheum 2012;64:2677-86.

17. Tan EM, Cohen AS, Fries JF, Masi AT, McShane DJ, Rothfield NF, et al. The 1982 revised criteria for the classification of systemic lupus erythematosus. Arthritis Rheum 1982;25:1271-7.

18. Team R. Rstudio: Integrated development for R. Boston, MA: RStudio Inc.; 2015.

19. Sultan SM, Begum S, Isenberg DA. Prevalence, patterns of disease and outcome in patients with systemic lupus erythematosus who develop severe haematological problems. Rheumatology 2003;42:230-4.

20. Gupta A, Weitzman S, Abdelhaleem M. The role of hemophagocytosis in bone marrow aspirates in the diagnosis of hemophagocytic lymphohistiocytosis. Pediatr Blood Cancer 2008;50:192-4.

21. Inoue T, Takeda T, Koda S, Negoro N, Okamura M, Amatsu K, et al. Differential diagnosis of fever in systemic lupus erythematosus using 
discriminant analysis. Rheumatol Int 1986;6:69-77.

22. Sarkissian T, Beyene J, Feldman B, McCrindle B, Silverman ED. Longitudinal examination of lipid profiles in pediatric systemic lupus erythematosus. Arthritis Rheum 2007;56:631-8.

23. Park YW, Kee SJ, Cho YN, Lee EH, Lee HY, Kim EM, et al. Impaired differentiation and cytotoxicity of natural killer cells in systemic lupus erythematosus. Arthritis Rheum 2009;60:1753-63.

24. Cuadrado MJ, Marubayashi M, Ortega C, Fernandez-Arcas N, Garcia-Cozar F, Pena J, et al. Relationship of IL-2, IL-2R (CD25+), soluble IL-2R and IL-4 with disease activity in SLE patients. Lupus 1993;2:257-60.
25. Swaak AJ, Hintzen RQ, Huysen V, van den Brink HG, Smeenk JT. Serum levels of soluble forms of $T$ cell activation antigens CD27 and CD25 in systemic lupus erythematosus in relation with lymphocytes count and disease course. Clin Rheumatol 1995;14:293-300.

26. Eloseily EMA, Minoia F, Crayne CB, Beukelman T, Ravelli A, Cron $R Q$. Ferritin to erythrocyte sedimentation rate ratio: simple measure to identify macrophage activation syndrome in systemic juvenile idiopathic arthritis. ACR Open Rheumatol 2019;1:345-9.

27. Borgia RE, Gerstein M, Levy DM, Silverman ED, Hiraki LT. Features, treatment, and outcomes of macrophage activation syndrome in childhood-onset systemic lupus erythematosus. Arthritis Rheumatol 2018;70:616-24. 